\title{
Advances in High Resolution Helium Ion Microscope (HIM) Imaging
}

\author{
Colin Sanford*, John Notte*, Larry Scipioni*, Shawn McVey*, Ray Hill*, Sybren Sijbrandij*,
} Lou Farkas*

*Carl Zeiss SMT, Inc, One Corporation Way, Peabody, MA, 01960

Recent publications have explored the resolution performance of a Helium Ion Microscope (HIM) relative to conventional Scanning Electron Microscopes (SEMs) and Gallium Focused Ion Beams (Ga-FIBs) [1-3]. Theoretical analysis of the achievable image resolution indicates that resolution superior to SEMs and Ga-FIBs should be achievable with HIMs due to its small probe size, high secondary electron yield, smaller interaction volumes, high ratio of SE1 to SE2, and other factors relative to SEMs and Ga-FIBs. In practice we find that the secondary electron resolution from a HIM is indeed superior to conventional SEMs and Ga-FIBs.

During our work with the HIM we have explored several different contrast mechanisms and have performed high resolution imaging in both a conventional top surface imaging mode, as well as a transmission mode. Typically top surface imaging is accomplished using ion induced secondary electrons, while transmission mode is accomplished by using transmitted helium ions which upon impact with a conversion surface are converted into electrons which can then be detected by a standard secondary electron detector. Modeling results of the helium beam interaction with the sample show that image resolution well below $1 \mathrm{~nm}$ is expected for top surface secondary electron imaging with the HIM, consistent with our experimental results. An often overlooked advantage of the HIM is that the ultra high resolution images are obtainable at relatively long physical working distances. This is a direct result of the small convergence angle, high brightness, and low intrinsic energy spread of the helium ion source. Some of the best images to date have been obtained with a free working distance of $6 \mathrm{~mm}$. This long working distance allows for other detectors to be included in the instrument and also allows the sample to be relatively unconstrained in size while still being able to tilt. Amazingly, high quality images are obtainable at working distances greater than $500 \mathrm{~mm}$. This paper will present a variety of ultra high resolution imaging results that demonstrate the use of transmission ion mode as well as top surface secondary electron mode. Unique attributes of the HIM such as its large depth of focus, large free working distance, and extreme surface sensitivity will be presented. Recent progress towards increasing secondary electron collection efficiency and other recent advancements such as variable accelerating voltage capability on the Orion ${ }^{\mathrm{TM}}$ Helium Microscope will be discussed. Variable accelerating voltage allows the HIM to be used effectively for voltage contrast applications, and explore other energy sensitive contrast mechanisms. Due to the significantly reduced damage-to-information threshold associated with a helium based ion beam relative to a Ga-FIB, voltage contrast on much smaller structures is possible.

\section{References}

[1] D.C. Joy et al., Proc. SPIE 6518: 65181I (2007)

[2] K. Inai et al., J. Electron Microsc., 56163 (2007) 1.

[3] D. Cohen-Tanugi and N. Yao, J. Appl. Phys, 104 (2008) 063504-1 


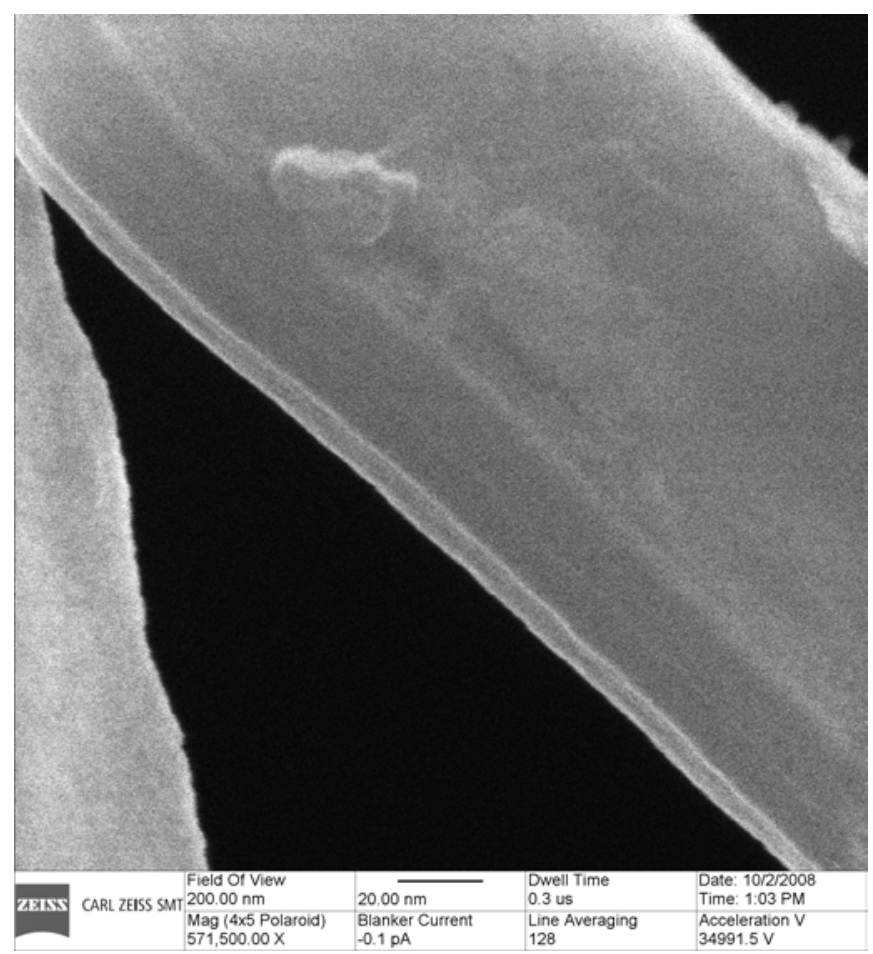

Fig. 1. Top surface secondary electron image of asbestos fiber on holey carbon grid. FOV = $200 \mathrm{~nm}$.

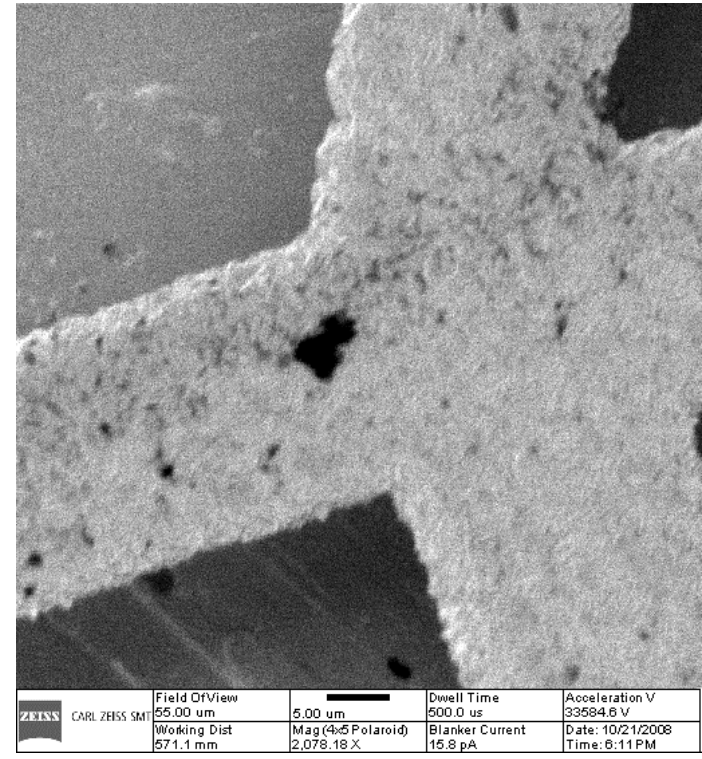

Fig. 2. HIM image demonstrating exceptionally long working distance; $\mathrm{WD} \sim 570 \mathrm{~mm}$. FOV $=55 \mu \mathrm{m}$.

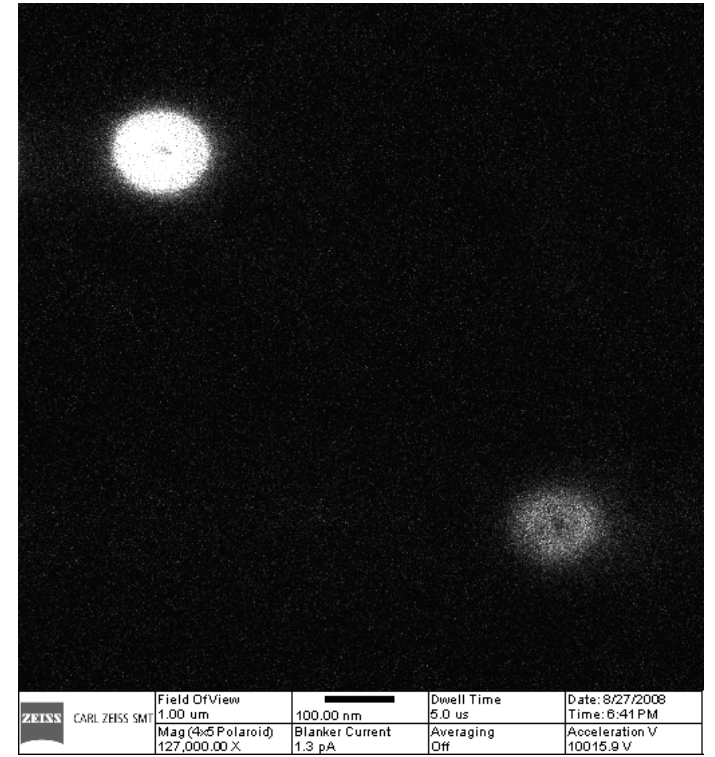

Fig. 3. Secondary electron image from HIM demonstrating voltage contrast between two $150 \mathrm{~nm}$ contacts. 